\title{
A technology-based nutritional education intervention to increase fruit and vegetables consumption in university students: findings from a randomised controlled study
}

\author{
Alyson Hill, Amin Hafiz and Alison Gallagher \\ Ulster University, Coleraine, United Kingdom
}

\begin{abstract}
Background: The transition to University is recognised as a vulnerable period for many young adults with the establishment of unhealthy eating behaviours. Evidence indicates that diets are low in fruit and vegetables, high in confectionary and fast food therefore, having the potential to develop obesity and long-term health implications. This RCT aimed to investigate the impact of providing 5 portions of fruit and vegetables $(\mathrm{F} \& \mathrm{~V})$ daily on consumption and plasma measurements. In addition to investigate whether intake was enhanced by nutritional education delivered using Facebook (FB)
\end{abstract}

Methods: Sixty full-time university students ( 36 females, 24 males) were randomly assigned to one of 3 groups: $F \& V$ received five portions $(80 \mathrm{~g})$ of fruit and vegetables daily for 4 weeks; $\mathrm{F} \& V+F B$ received as for $\mathrm{F} \& \mathrm{~V}$ group and in addition nutrition messages using FB; Control group maintained their usual diet. Compliance with F\&V consumption was determined by analysis of vitamin $\mathrm{C}$ and carotenoid levels and in addition recorded intake and returned all uneaten F\&V. Level of engagement on FB page was categorized according to interaction: highly active, somewhat active (interacts $>$ once per month) or minimally active (interacts

Results: Participants were aged 24.4 (SD 3.7) years and mean BMI of 25.4 (SD 4.7) kg/m². Study groups did not differ by age, however they did differ by gender (24 male and 36 female, $P=0.003)$, BMI $(P=<0.039)$ and TEI $(P=<0.041)$. The number of portions of FV consumed increased in both intervention groups $(P=<0.001)$ and showed that $65 \%$ of participants increased intake of FV in both intervention groups $(P=<0.001)$ from baseline to end of study of $4.6(0.6) F \& V$ group, $4.7(0.4) F \& V+F B$ group. Mean change in serum $\beta$ carotene was significantly higher for both intervention groups than the control group at the end of the four-week intervention period. However, the provision of F\&V with or without FB did not have any enhanced effect on FV consumption and was not sustained at 3 weeks after the end of the intervention. Of those 20 participants in the $F \& V+F B$ group, $70 \%$ engaged with FB regularly with $35 \%$ classified as highly active, $15 \%$ moderately active and $50 \%$ minimally active.

Conclusions: This study showed an increased consumption from 1.6 to 4.6 portions fruit and vegetables daily over the 4 week study period and showed an increase in vitamin $\mathrm{C}$ and retinol levels. However, providing additional nutrition education via FB was insufficient to increase consumption.

\section{Conflict of Interest}

No conflict 